\title{
Formação na Graduação em Psicologia no SUS: Percepções de Psicólogos Residentes
}

\section{Training in Graduation in Psychology in SUS: Perceptions of Residents in Psychology \\ Entrenamiento en Graduación en Psicología En SUS: Percepciones de Psicólogos Internos Residentes}

\author{
Patrícia Matte Rodrigues \\ Dorian Mônica Arpini \\ Camila Almeida Kostulski ${ }^{1}$ \\ Universidade Federal de Santa Maria (UFSM)
}

\begin{abstract}
Resumo
Introdução: O estudo teve o objetivo de abordar a formação acadêmica de psicólogos, vinculados a Residências Multiprofissionais, para atuação no Sistema Único de Saúde (SUS).

Método: Para tanto, realizou-se uma pesquisa qualitativa com psicólogos residentes que pertenciam às áreas de ênfase Saúde da Família e Comunidade e Atenção Básica em Saúde Coletiva de dois Programas de Residência da região Sul do Brasil. Para a coleta dos dados, foram realizadas entrevistas semiestruturadas, analisadas por meio da análise de conteúdo temática. Resultados e discussões: Evidenciaram-se movimentos de aproximação dos cursos de graduação em psicologia com as temáticas do SUS, Saúde Coletiva e Políticas Públicas, o que parece proporcionar certa ampliação do olhar dos psicólogos para esse contexto de atuação. Conclusões: As considerações finais indicam potencialidades e impasses na formação da graduação em psicologia para o trabalho no SUS e na atenção básica.

Palavras-chave: formação profissional, políticas públicas, Sistema Único de Saúde; psicólogo, residências em saúde
\end{abstract}

\begin{abstract}
Introduction: This study aimed to approach the academic training of psychologists linked to Multiprofessional Residence for the Brazilian National Health Service (SUS). Method: Therefore, there was conducted a qualitative research with residents in psychology whose fields of study were Community and Family Health and Primary Healthcare in Public Health - these were part of two Residence Programs located in the south of Brazil. For data collection, it was used semi-structured interviews, which analysis was based on the thematic content analysis. Results and discussions: There were perceived moves of approaching among undergraduate psychology courses with themes such as SUS, Public Health, and Public Policies, which seems to promote proximity of psychologists to this area of practice. Conclusions: Final considerations indicate potentialities and impasses in the training of undergraduate psychology for work in SUS and primary care.
\end{abstract}

Keywords: professional training, public policies, Sistema Único de Saúde, psychologist, health residences

\section{Resumen}

Introducción: La investigación tuvo como objetivo abordar el entrenamiento académico de psicólogos, vinculado a Residencias Multiprofesionales, para trabajar en el Sistema Brasileño de Salud Pública (SUS). Método: Para eso, se hizo una investigación cualitativa con psicólogos internos residentes de las áreas de Salud de la Familia y la Comunidad y Atención Primaria en Salud Colectiva, de la región Sur de Brasil. Para la colección de los datos se hicieron entrevistas semiestructuradas, analizadas a través del análisis de contenido temático. Resultados y discusiones: Se evidencian movimientos de acercamiento de los cursos de grado en Psicología a las temáticas como SUS, Salud Colectiva y Políticas Públicas, lo que indica una ampliación de la mirada de los psicólogos hacia ese contexto de prácticas. Conclusiones: Las consideraciones finales indican potencialidades e impases en la formación de psicología de pregrado para el trabajo en el SUS y en la atención primaria.

Palabras clave: entrenamiento profesional, políticas públicas, Sistema Único de Saúde, psicólogo, residencias de salud

\footnotetext{
${ }^{1}$ Endereço para contato: Rua dos Andradas, 1465, ap. 507, Centro, Santa Maria, RS, Brasil. CEP: 97010-033. Telefone: (55) 99961-3550. E-mail: camila.ak@hotmail.com
} 


\section{Introdução}

Em meio a um cenário de discussões sobre a formação de profissionais para o trabalho nas políticas públicas de saúde no Brasil, os cursos de graduação em Psicologia têm sido incentivados a realizar mudanças em sua organização curricular. Estabelecida como uma profissão que, durante muito tempo, trabalhou por meio de um viés clínico, individual, atendendo uma clientela elitizada, a Psicologia hoje se vê diante da ampliação de suas áreas tradicionais de atuação e do comprometimento acerca da reflexão de seus saberes (Dimenstein, 2001; Yamamoto, 2003; C. Silva \& Yamamoto, 2013).

Uma das áreas que têm proporcionado um importante espaço, nos seus três níveis de atenção, para o trabalho do psicólogo é o Sistema Único de Saúde (SUS), que é considerado o arranjo organizacional responsável pela efetivação da política de saúde e uma das principais inovações da reforma do Estado Brasileiro (Dimenstein, 2001). Contudo a literatura assinala que essa nova conformação da profissão tem gerado um importante debate a respeito da formação e atuação voltada para essa área, e em especial ao trabalho na atenção básica (Dimenstein \& Macedo, 2010; Spink \& Matta, 2010; Ferreira, 2011; Azevedo, Tatmatsu \& Ribeiro, 2011; Davi, Dominguez, Araújo, \& Ribeiro, 2016).

Em pesquisas desenvolvidas sobre a atuação de psicólogos nesse contexto, autores verificaram a presença do modelo de clínica privada, com enfoque individual, curativo e voltado para a psicoterapia de longa duração (Dimenstein, 2001; Böing \& Crepaldi, 2010; Dimenstein \& Macedo, 2010; Spink \& Matta, 2010). Não por acaso, Nepomuceno (2014) afirma que a psicologia tem atuado de forma limitada e mesmo incipiente nas políticas públicas de saúde. Sobre essa questão, estudos indicam que, apesar da ampliação dos campos de trabalho, o psicólogo continua a desenvolver atividades muito semelhantes, independentemente do contexto em que se encontra (Bastos, Gondim, \& Borges-Andrade, 2010; Davi et al., 2016).

No entanto a forma como o SUS aborda as questões da saúde, ou seja, a partir de um olhar ampliado sobre os diferentes aspectos de vida da população, demanda um profissional capaz de desenvolver práticas condizentes com essa perspectiva de cuidado (Reis \& Guareschi, 2013). As reflexões das autoras se assemelham ao que é discutido por Dimenstein e Macedo (2010), ao afirmarem que o trabalho no SUS implica uma formação que considere diferentes aspectos da vida do indivíduo, um conhecimento interdisciplinar e o compromisso com o desenvolvimento de práticas voltadas às necessidades da população. Esse aspecto foi destacado também por Rocha, Almeida e Ferreira (2016), ao enfatizarem a pluralidade de ações que devem estar presentes na prática dos psicólogos na atenção básica, como: o reconhecimento do território, ações grupais, suporte técnico, planejamento em equipe, preceptorias e consultorias, entre outras.

No que diz respeito ao nível de atenção básica, considerada porta de entrada do sistema público de saúde (Starfield, 2002), a literatura confere destaque à necessidade de aprofundamento de questões relacionadas à formação do psicólogo, para que não haja a simples transposição de ações do modelo clínico tradicional ao nível primário de atenção à saúde (Soares, 2005; Böing \& Crepaldi, 2010; Rocha et al., 2016; Rosa \& Silva-Roosli, 2019). Nepomuceno (2014) destaca um processo de reconfiguração que vem sendo realizado no âmbito da saúde, com a criação de novos ambientes de trabalho, tais como os Núcleos de 
Apoio à Saúde da Família (NASF) e as Residências Multiprofissionais em Saúde (RMS), que possibilitam uma atuação diferenciada do psicólogo no sistema público de saúde. Contudo o autor ressalta que a entrada da psicologia nesses cenários ainda tem evidenciado um despreparo da categoria para desenvolver um trabalho em sintonia com os imperativos propostos pelo atual sistema público de saúde.

Desse modo, um dos desafios que o psicólogo enfrenta ao trabalhar no SUS é o de uma formação mais próxima de seus princípios e das necessidades de seus usuários (Spink \& Matta, 2010). Mas essa não é uma preocupação apenas desse núcleo profissional. A literatura evidencia que a formação de profissionais na área da saúde, na qual também se encontra a Psicologia, apresenta ainda um ensino clássico, tecnicista e preocupado com a sofisticação dos procedimentos, mostrando-se distante da realidade social (Dimenstein \& Macedo, 2010; Ceccim \& Feuerwerker, 2004). Nesse sentido, a necessidade de mudanças na formação em saúde tem sido uma questão bastante debatida no país, provocando movimentos nos cursos de graduação. Essas propostas formativas partem do entendimento de que a formação é uma das questões centrais no que diz respeito à transformação das práticas profissionais mais alinhadas às políticas de saúde (Biscarde, Pereira-Santos, \& Silva, 2014).

Em relação à formação dos psicólogos, as intensas discussões sobre mudanças no ensino acadêmico ganharam força com a publicação das Diretrizes Curriculares Nacionais (DCNs) para os cursos de Psicologia, no ano de 2004, as quais foram fortalecidas pelas DCNs publicadas em 2018. O primeiro documento sugere novos caminhos para a formação desse profissional, a partir de uma concepção de ensino generalista, que incentiva uma maior flexibilização dos desenhos curriculares (Ceccim \& Carvalho, 2005) e que apresenta como uma de suas ênfases a atenção à saúde. O segundo apresenta propostas que contemplam a diversidade de possibilidades no que diz respeito tanto às áreas de atuação quanto aos fundamentos epistemológicos e metodológicos da formação em Psicologia. Consideram fundamental a inserção de ênfases curriculares voltadas para as Políticas Públicas, as quais contemplem sua gestão, fundamentos e prática, reconhecendo, assim, a pluralidade da Psicologia e assegurando, nos currículos, conhecimentos nas áreas da saúde, assistência social, educação, trabalho, entre outras (Resolução n. 597, 2018).

As DCNs propõem, dessa forma, a articulação de conteúdos e o desenvolvimento de competências e habilidades que buscam despertar no acadêmico a compreensão da importância da integração do ensino, dos serviços de saúde e da comunidade, como meio de aproximar o estudante da realidade dos locais de atuação (Abdalla, Batista, \& Batista, 2008). Assim, movimentos de mudanças no ensino acadêmico têm produzido importantes reflexões sobre o profissional que os cursos de psicologia vêm formando para atuar nas políticas públicas de saúde.

Nesse sentido, cabe mencionar a formação médica, a qual vem passando por mudanças de paradigmas do currículo, apontando algumas alterações que podem ser importantes para os desafios que a Psicologia como profissão da saúde também encontra. Podemos tomar como exemplo, considerando sua relevância e o fato de estar sendo utilizado por muitos países em vários continentes, o CanMEDs (Frank, Snell, \& Sherbino, 2015). Este surgiu no Canadá, no início dos anos 1990, e vem sendo atualizado regularmente, encontra-se atualmente na sua terceira edição, de 2015. Sua estrutura está organizada em sete grupos 
de competências, as quais expressam os papéis médicos: especialista, comunicador, colaborador, líder, advogado, acadêmico e profissional. Estas devem estar interligadas e evidenciar um profissional com competência para comunicar pacientes e familiares, falar em linguagem clara sobre os problemas, entender e reconhecer o ponto de vista do paciente, ser um profissional com capacidade de liderança e engajamento, envolvido com o sistema de saúde. Deve ser também um profissional capaz de colaborar com os demais colegas que atuam na saúde, integrando-se a equipes. Além disso, necessita compartilhar seus conhecimentos para influenciar as comunidades e as populações, considerando os vários determinantes em saúde e a compreensão da própria comunidade. Nesse sentido, sua identidade central nesse processo é ser um profissional dedicado à saúde e a seus cuidados (Frank et al, 2015).

É importante ressaltar que uma das estratégias encontradas pelo Governo Federal para aprimorar o conhecimento dos profissionais da área da saúde e capacitá-los para atuação condizente com os princípios do SUS são as Residências Multiprofissionais em Saúde (Lei n. 11.129, 2005). Desenvolvidas em regime de dedicação exclusiva, possuem carga horária de 60 horas semanais, divididas em atividades práticas e teórico-reflexivas, e contam com supervisão docente assistencial (Resolução n. 2, 2012).

A partir dessa perspectiva, procurou-se compreender se a formação acadêmica de psicólogos que se encontram vinculados a Programas de Residência Multiprofissional em Saúde contemplou as exigências para um trabalho no SUS, mais especificamente, neste estudo, no contexto da atenção básica.

\section{Método}

O presente estudo constitui-se de uma pesquisa qualitativa, de caráter exploratório. A pesquisa qualitativa tem o intuito de explorar as diferentes representações e ideias sobre o assunto em questão, de modo a descobrir a variedade de pontos de vista sobre a temática estudada, além do que fundamenta e justifica estes diferentes pontos de vista (Minayo, 2006, 2014; Gaskell, 2005).

Os participantes do estudo foram oito psicólogos residentes, vinculados a dois Programas de Residência Multiprofissional em Saúde (RMS) de duas instituições de ensino e pesquisa em saúde da região sul do Brasil. Os participantes pertenciam às áreas de ênfase Saúde da Família e Comunidade e Atenção Básica em Saúde Coletiva, e, como critério de inclusão, deveriam estar cursando o segundo ano da RMS. Tal critério foi utilizado considerando que os residentes já teriam a experiência prática de um ano nos campos de trabalho, o que possibilitaria melhor atender aos objetivos do estudo.

Contudo, por questões éticas, optou-se por não realizar a identificação do Programa de RMS ao qual os participantes estavam vinculados, visto que essa informação poderia acarretar a identificação do residente, devido às especificidades do estudo. Essa escolha está amparada na Resolução n. 510 (2016), do Conselho Nacional de Saúde, a qual dispõe sobre as normas aplicáveis a pesquisas em Ciências Humanas e Sociais. 
Tabela 1

Características dos Participantes da Pesquisa

\begin{tabular}{ccccccc}
\hline Participante & Sexo & Idade & $\begin{array}{c}\text { Instituição de } \\
\text { Ensino Superior }\end{array}$ & $\begin{array}{c}\text { Ano de conclusão } \\
\text { da graduação }\end{array}$ & $\begin{array}{c}\text { Outra } \\
\text { especialização }\end{array}$ & $\begin{array}{c}\text { Experiência } \\
\text { profissional }\end{array}$ \\
\hline P1 & F & 26 & Privada & 2014 & Não & Não \\
P2 & M & 31 & Privada & 2014 & Não & Sim \\
P3 & F & 25 & Privada & 2014 & Sim & Não \\
P4 & F & 25 & Pública & 2014 & Não & Não \\
P5 & M & 24 & Privada & 2013 & Não & Sim \\
P6 & F & 24 & Pública & 2014 & Não & Não \\
P7 & F & 31 & Privada & 2011 & Sim & Sim \\
P8 & F & 26 & Pública & 2013 & Não & Não \\
\hline
\end{tabular}

Fonte: Elaborada pelas autoras.

Primeiramente, realizou-se o contato com as Coordenações dos dois Programas de RMS para apresentação do projeto de pesquisa e solicitação do Termo de Autorização Institucional. Após o aceite por parte das Coordenações, o projeto foi submetido ao Comitê de Ética em Pesquisa da Instituição de Ensino Superior na qual as pesquisadoras estão vinculadas, por meio da Plataforma Brasil, tendo sido aprovado pelo Certificado de Apresentação para Apreciação Ética (CAAE) sob número 56594116.2.3001.5530.

O contato com os residentes foi mediado pelas respectivas coordenadoras dos Programas de RMS. A técnica utilizada foi a de entrevistas individuais semiestruturadas, as quais foram agendadas de acordo com a disponibilidade dos participantes, ocorrendo na sede das instituições envolvidas. As entrevistas foram gravadas mediante a permissão dos participantes, por meio da assinatura do Termo de Consentimento Livre e Esclarecido (TCLE).

Vale ressaltar que o estudo atendeu a todas as exigências da ética em pesquisa, conforme a Resolução n. 466 (2012), bem como a Resolução n. 510 (2016). Procurando manter o anonimato dos participantes, ocultou-se qualquer informação que pudesse identificá-los. Dessa forma, as falas serão apresentadas com a letra $P$, seguidas do número que representava a ordem das entrevistas (P1, P2 . . .), não havendo indicação alguma de qual Programa de Residência Multiprofissional pertencia o participante.

As entrevistas foram orientadas a partir de tópicos-guia estabelecidos de acordo com os objetivos do estudo, são eles: 1) Formação Acadêmica; 2) Experiência Profissional e 3) Percepção sobre o Trabalho na RMS. Para a análise dos dados oriundos das entrevistas, utilizou-se a análise de conteúdo (Bardin, 2010), com enfoque na modalidade de análise de conteúdo temática (Minayo, 2014).

\section{Resultados e Discussão}

\section{Movimentos da Formação em Psicologia: a Aproximação com o Contexto do SUS}

No que diz respeito à formação na graduação dos psicólogos residentes entrevistados, a maioria assinalou a presença de um número significativo de disciplinas, as quais abordavam temáticas relativas ao SUS, Saúde Coletiva e/ou Políticas Públicas. Tal questão foi entendida 
pelos participantes como um ponto positivo de sua formação, visto que teria proporcionado uma aproximação com o contexto público de saúde, trabalhando diferentes aspectos da atuação do psicólogo:

... tem muitas disciplinas de políticas públicas, saúde coletiva, enfim, de políticas públicas de saúde, políticas públicas no geral assim para poder também dar conta das políticas de educação, de assistência social . . é uma formação bem importante, é um curso [de graduação] que propicia que a gente tenha contato com várias dimensões, de aspectos de trabalho, das práticas do psicólogo. (Participante 1, comunicação pessoal, 6 de maio, 2016)

Tive disciplinas de saúde coletiva e políticas públicas, de psicologia da saúde, que era voltado mais para as políticas públicas específicas da saúde, de como a psicologia atua minimamente. . . ela fazia uma certa relação com a psicologia comunitária, do trabalho com as áreas da saúde. (Participante 4, comunicação pessoal, 17 de maio 5, 2016)

As falas dos participantes indicam mudanças que têm sido incentivadas nos currículos de graduação em psicologia após a publicação das DCNs, que, além de outros aspectos, estaria propondo uma formação de cunho generalista que proporciona a inclusão de saberes e práticas voltadas para a atuação do psicólogo nas políticas públicas de saúde (Reis \& Guareschi, 2013). Desse modo, parece ser possível inferir que os cursos de psicologia, nos quais os participantes concluíram suas graduações, contêm disciplinas que buscam propiciar certa ampliação do olhar dos acadêmicos para além do modelo clínico tradicional. Nesse sentido, compreende-se que o ensino da psicologia estaria mais sensível aos múltiplos cenários de atuação do psicólogo, adequando suas grades curriculares às novas demandas. Sendo assim, em concordância com Soares (2005), compartilhamos da noção de que as instituições formadoras devem repensar o profissional psicólogo que desejam formar, considerando a mudança dos paradigmas que as têm orientado até o momento.

Cabe ressaltar que duas instituições de ensino superior tiveram destaque nas entrevistas. A primeira delas, citada por (P1) e (P5), é uma instituição privada de ensino que, de acordo com os psicólogos, realizou mudanças em seu currículo entre os anos de 2009 e 2010, estabelecendo duas ênfases de formação, uma delas com enfoque em políticas públicas, e a outra, em processos clínicos. Segundo os participantes, as duas ênfases proporcionavam certa abertura para diferentes disciplinas e estágios, não se constituindo como um currículo fechado, conforme relatado por (P1) ". . . além da gente ter uma formação, claro mais nesse formato digamos mais clínico, enfim das linhas teóricas, de abordagem da psicologia, tem muitas disciplinas de políticas públicas" (Participante 1, comunicação pessoal, 6 de maio, 2016). É importante salientar que a residente realizou a graduação com ênfase em políticas públicas, enquanto (P5) em processos clínicos. Contudo, o participante também aponta para a flexibilidade assinalada anteriormente:

. . como eu peguei essa etapa de transição do curso, eu acabei tendo que optar pela ênfase clínica para conseguir me formar no tempo ... mas isso é bem flexível, tanto que o meu TCC, a minha prática toda era mais voltada mais para a saúde pública apesar da ênfase ser clínica. (Participante 5, comunicação pessoal, 20 de junho, 2016)

Já a segunda Instituição é uma Universidade Pública, onde o curso de Psicologia formou sua primeira turma no ano de 2012. As participantes (P4) e (P8) realizaram sua graduação na 
Instituição. Nesse sentido, assinalaram que o curso apresenta grande enfoque para a área da saúde, não apenas com a presença de disciplinas voltadas para a temática, mas com o desenvolvimento de estágios, projetos de extensão e a aproximação com serviços públicos de saúde, o que foi valorizado pelas psicólogas residentes. Além disso, (P4) apontou essa característica do curso como fundamental para a decisão de realizar a RMS: " . . eu considero que a minha graduação foi bem importante na minha decisão assim de fazer Residência, acho que eles fizeram um start bom ... " (Participante 4, comunicação pessoal, 17 de maio, 2016).

Dito isto, além da importância da inclusão de disciplinas voltadas para a Saúde Coletiva, Políticas Públicas e SUS, na busca por uma formação mais alinhada às atuais demandas dos espaços de atuação do psicólogo, considera-se positivo que a presença de tais disciplinas, assim como: psicologia comunitária, social, da saúde, psicologia do trabalho e institucional, que também foram assinaladas pelos participantes como importantes no que diz respeito a uma formação ampliada e reflexiva, tenham estimulado o interesse dos acadêmicos para o contexto do SUS e da própria RMS.

De certo modo, essa questão é referida por uma das psicólogas que entende ser uma particularidade do seu curso de graduação apresentar uma proposta mais alinhada as questões do SUS, já que se trata de um curso novo, mas apresenta um contraponto:

$E$ É eu acho que os cursos mais antigos tinham mais o caráter de clínica então . . acho que quando a psicologia entrou na $X$ [universidade da graduação] eles tiveram muito esse cuidado de apresentar a clínica, mas como um ponto do fazer do psicólogo, que tinha muito mais essas outras possibilidades. (Participante 8, comunicação pessoal, 20 de agosto, 2016)

A colocação da participante de que o contexto clínico é "um ponto do fazer do psicólogo", mas não o único, parece despontar como uma possível consequência das atuais mudanças nos currículos que consequentemente visam operar transformações no modo de atuar dos profissionais da psicologia. Assim, quando Dimenstein (2001) destaca que os psicólogos precisam incorporar uma nova compreensão de prática profissional, rompendo com ações isoladas e com uma identidade profissional hegemonicamente ligada à do psicoterapeuta, a compreensão apresentada pela residente parece revelar a construção de um pensamento alinhado à ideia desenvolvida pela referida autora.

Na contramão desse movimento de mudanças nos currículos exposto até o momento, apresentam-se os relatos de alguns participantes, que durante sua graduação vivenciaram currículos mais tradicionais, com poucas disciplinas voltadas para um trabalho no SUS:

. . . cheguei a ter duas cadeiras, três no máximo, que era aquelas cadeiras de psicologia comunitária enfim, mas sim tive, pouco, porque a formação da X [universidade da graduação] ela é muito voltada para clínica privada mesmo, e agora que está se abrindo mais para, até por uma questão de mercado. (Participante 2, comunicação pessoal, 6 de maio, 2016)

. . a minha turma era a última turma do currículo antigo, então eu peguei a última turma que ainda era do currículo antigo. O currículo novo, sim, está bem diferente, tem bem essa coisa da saúde como, e da política pública como norte. (Participante 6, comunicação pessoal, 5 de agosto, 2016) 
... eu sei que hoje em dia a X [universidade] modificou o currículo, começou a dar atenção mais para essas disciplinas de políticas públicas. Na minha época, como eu sempre gostei, eu ia atrás então, as eletivas que a gente tinha eu ia para o Serviço Social, que tinha saúde coletiva, tinha SUS, porque no currículo da psicologia não abordava. (Participante 7, comunicação pessoal, 26 de agosto, 2016)

As falas dos residentes acabam por reforçar discussões presentes na literatura sobre o ensino oferecido por cursos de psicologia no país (Dimenstein, 2001; Romagnoli, 2006; Spink \& Matta, 2010; Azevedo et al., 2011). Tais estudos apresentam diversas críticas a formação dos psicólogos que ainda hoje mantém algumas lacunas no que diz respeito ao investimento em temáticas voltadas principalmente para o campo da saúde. Contudo, a formação em psicologia vem passando por mudanças importantes, de uma formação com enfoque na clínica para uma perspectiva mais social e uma opção pela saúde pública, podendo se inferir, tal como apontado pelo participante (P2), que o mercado de trabalho seria um elemento importante para essas mudanças.

Em material organizado pelo Conselho Federal de Psicologia [CFP] (2010), que aborda a atuação de psicólogos na atenção básica, estes apontaram para o despreparo diante dos desafios políticos, sociais e profissionais do contexto público. Além disso, os psicólogos relataram uma formação em descompasso com o trabalho exigido na atenção básica o que, segundo eles, resultava em um aprendizado que ocorria na prática. As discussões construídas nesse material mostram a relevância que o ingresso do psicólogo no cenário da saúde tem apresentado, impulsionando novos estudos, vistos como necessários, sobre a importante interface da psicologia com o SUS, a Saúde Coletiva e a atenção básica.

Seja pelo incentivo das DCNs ou pela crescente inserção de psicólogos em cenários como NASF, atenção básica, Centro de Atenção Psicossocial (CAPS) e Sistema Único de Assistência Social (SUAS), os cursos de graduação de (P2), (P6) e (P7) sofreram mudanças curriculares, e apresentam segundo os entrevistados, propostas de ensino que buscam acompanhar as novas conjunturas da formação em psicologia. Cabe destacar que a questão apontada por (P7) de sua procura por disciplinas que oferecessem certa aproximação com o SUS, Políticas Públicas, e de certa forma com a atenção básica, também se fez presente na fala de outros participantes. Seus relatos mostraram tanto uma procura por disciplinas em outros cursos de graduação, quanto o envolvimento com projetos de extensão, cursos, oficinas, palestras e estágios que proporcionassem conhecimentos teóricos e práticos sobre o trabalho no sistema público de saúde. Entende-se, dessa forma, que os participantes do estudo já vinham trilhando caminhos próximos ao nível da atenção básica e do SUS, o que pode demonstrar um interesse despertado na graduação e que vem sendo consolidado junto a RMS.

Nesse sentido, o que se percebe é que os movimentos que estão sendo feitos pelos cursos de graduação para o desenvolvimento de propostas de ensino mais alinhadas às questões do SUS, ainda não atendem satisfatoriamente às expectativas dos acadêmicos, segundo referiram os psicólogos entrevistados. A fala do participante (P2) corrobora com esse entendimento, apontando para um processo ainda em construção: “ . . a acho que vem passando por uma modificação, mas isso vai depender muito da gente assim, das nossas experiências, da gente poder avaliar e monitorar isso que a gente está passando e exigir mudanças até de formação . . . " (Participante 2, comunicação pessoal, 6 de maio, 2016). 
Desse modo, pode-se inferir que a graduação em Psicologia se encontra num momento de transição quanto a formação para a atuação dos psicólogos nas políticas públicas, apresentando algumas dificuldades para inserir conteúdos fundamentais para o trabalho na saúde, questão que foi percebida quando os participantes se depararam com o campo de prática da RMS. Nesta mesma direção, Pasini (2010) faz uma indagação interessante, ao questionar o tipo de profissional que está sendo formado para atuar na saúde. Ainda que em seu texto a autora aborde principalmente o âmbito da RMS pode-se fazer a articulação de suas ideias com as do presente estudo, uma vez que se entende que esse questionamento possa vir a produzir importantes reflexões para a formação acadêmica dos psicólogos. Nesse sentido, em concordância com a autora, pontua-se que o ensino de Psicologia parece ainda não dar conta de formar profissionais qualificados para lidar com os desafios propostos pelo SUS, apesar das mudanças significativas que vêm sendo realizadas. Nessa mesma perspectiva, Davi et al. (2016) chamam atenção para a ausência de inovações que se apliquem efetivamente ao setor público, existindo ainda certa incompatibilidade entre a realidade dos serviços e as teorias e técnicas aprendidas na formação universitária.

Outro aspecto que deve ser salientado em relação à formação dos entrevistados foi a presença de professores que se mostraram importantes em seu percurso acadêmico. Os depoimentos referem que esses docentes foram fundamentais para aproximar os psicólogos do contexto do SUS:

... é que assim, a gente tem professores bem identificados com a questão da saúde coletiva sabe, enfim, pelo SUS, as políticas públicas né, e aí a gente tem lá esses professores, assim é bem marcante assim a realização do Ver-SUS né ... (Participante 1, comunicação pessoal, 6 de maio, 2016).

... um dos professores meus, ele era da rede assim, dos CAPS e tudo mais. Então acho que isso ajudou bastante ter. Um outro professor meu era na época da $4 \underline{a}$ Coordenadoria [de saúde] então acho que muito também por esses professores trazendo sabe e das leituras ... (Participante 3, comunicação pessoal, 10 de maio, 2016).

Estes relatos permitem analisar uma questão que parece ser central no processo de mudança dos cursos de graduação em Psicologia. Referimo-nos ao fato de que não bastaria apenas incluir as disciplinas voltadas para o SUS, na grade curricular, atendendo as DCN, mas sim, e de fato, avaliar como essas disciplinas estão sendo ministradas e trabalhadas pelos docentes responsáveis. Nas falas, os residentes referem-se a uma experiência na qual a identificação dos professores com as temáticas teria sido fundamental para despertar o interesse na área. Nesse sentido, uma das lacunas que poderia estar dificultando a consolidação desta área na formação, apesar da inserção de disciplinas vinculadas, seria exatamente esse aspecto, considerando que a ausência de uma tradição histórica consistente dos psicólogos nesse cenário, poderia fragilizar a tarefa docente, levando em conta que não haveria uma base de sustentação teórica e prática, podendo culminar numa certa inconsistência na apresentação do fazer da Psicologia no campo das políticas públicas, com professores que se sentem despreparados para o desafio de ministrar disciplinas nessas temáticas. Assim, ao tecer essa crítica, estaríamos reconhecendo que estamos abordando uma prática que demandou mudanças bastante significativas no fazer do psicólogo e que exige abertura e flexibilidade, para o trabalho multiprofissional, interdisciplinar, intersetorial, atuação em equipe, 
compartilhamento e discussão de casos, tomada de decisões, utilização de prontuários coletivos, ou seja, são vários aspectos que se redesenham e colocam em xeque nosso fazer mais tradicional (Rocha et al., 2016; Rosa \& Silva-Roosli, 2019; Rodrigues et al., 2017).

Retornando os depoimentos dos participantes, identificamos que estes mencionam a importância de os professores conhecerem o funcionamento dos serviços de saúde, seus desafios e suas potencialidades, pois entendem que esse fato torna o ensino mais próximo do que é vivenciado pelos profissionais no cotidiano de trabalho:

. . . eu acho muito ruim pensar a academia uma coisa descolada da prática . . . porque eu acho muito ruim professor que trabalha com essa temática, mas que nunca tiveram a inserção de verdade. Eu acho que precisa conhecer o trabalho, e também de produzir mudanças, de fortalecer o SUS, de estar inserida assim (Participante 6, comunicação pessoal, 5 de agosto, 2016).

Cabe destacar que o distanciamento entre o ensino acadêmico e os cenários de práticas, apontado pela residente (P6), ainda permanece como desafio. Entretanto, atualmente, as RMS constituem importantes estratégias para superação dessa distância que historicamente preocupa os idealizadores do sistema de saúde no país (Parisi \& De Antoni, 2014; Lima, 2015; R. B. Silva \& Carvalhaes, 2016).

Como exemplo disso, o estudo desenvolvido por Cezar, Rodrigues e Arpini (2015), o qual apresenta a inserção de psicólogas residentes no nível de atenção básica de um município do Rio Grande do Sul, assinala a importante aproximação que estava sendo construída entre a RMS e os cursos de graduação em psicologia do município, por meio da realização de estágios em unidades de saúde onde as residentes desenvolviam suas atividades. Nesse sentido, as autoras destacam a formação da Residência Multiprofissional como uma possível estratégia de mudança profissional dos psicólogos frente aos desafios propostos pelo contexto do sistema público de saúde.

Portanto, entende-se que pode ser um aspecto favorável para a graduação uma progressiva aproximação com a lógica de ensino da Residência Multiprofissional, como forma de promover uma articulação entre prática e formação, que se faz necessária diante dos diversos desafios, tanto na concretização de práticas quanto na construção de conhecimentos voltados às demandas do sistema público de saúde. Nesse sentido, pode-se estimar um impacto positivo do investimento das Residências Multiprofissionais na formação em nível de graduação, considerando o possível entrelaçamento entre esses níveis e o potencial das residências para repensar a lógica dos serviços e o fazer de cada núcleo profissional. Agrega-se ainda, o compromisso da residência com as premissas do Sistema Único de Saúde, o que em certa medida garante que a experiência do acadêmico junto aos programas de residência seja de fato uma aproximação a uma prática alinhada aos pressupostos do SUS.

\section{Considerações Finais}

A reflexão proposta pelo presente estudo identificou questões importantes na formação em psicologia para o trabalho no SUS e na atenção básica. Foi possível visualizar movimentos dos cursos de graduação, no sentido de oferecer um ensino mais alinhado à diversificação dos cenários de atuação dos psicólogos, em especial para a área da saúde, conforme 
propostas das DCNs. Esse importante movimento tem contemplado a inclusão de disciplinas voltadas para o SUS, Saúde Coletiva e Políticas Públicas, bem como a oportunidade de experiências práticas junto aos serviços públicos de saúde, o que parece possibilitar certa ampliação do olhar dos acadêmicos para as questões do SUS.

Contudo os depoimentos revelam um processo inacabado e que ainda precisa de investimento, tanto por parte das instituições de ensino superior como pelo Estado, com o estabelecimento de parcerias, o fortalecimento das políticas públicas em saúde e o compromisso com o processo de formação em saúde para o SUS. Uma formação que vislumbre não apenas práticas mais adequadas ao contexto de saúde pública, mas a ressignificação das questões que envolvem a produção da saúde.

Tendo em vista que a psicologia conquista cada vez mais espaço no contexto do SUS, é imprescindível refletir acerca da formação acadêmica que vem sendo oferecida nos cursos de psicologia em todo o país. Nesse sentido, o presente estudo traz importantes contribuições, revelando que, mais do que a inclusão das disciplinas, deve-se atentar para a forma como essas temáticas serão trabalhadas dentro dos cursos de psicologia, já que professores identificados com as propostas do SUS podem exercer um importante papel na aproximação dos acadêmicos com esse cenário.

Assim, esta pesquisa permitiu identificar potencialidades e impasses na formação em psicologia para o trabalho no SUS e na atenção básica junto a psicólogos que atuavam no cenário da Residência Multiprofissional em Saúde. Embora se reconheçam as contribuições advindas deste estudo, entende-se a necessidade da realização de novas pesquisas, em contextos diversificados, que possam potencializar as discussões já desenvolvidas sobre a formação acadêmica da psicologia para a atuação na saúde pública.

\section{Referências}

Abdalla, I. G., Batista, S. H., \& Batista, N. A. (2008). Desafios do ensino de psicologia clínica em cursos de psicologia. Psicologia: Ciência e Profissão, 28(4), 806-819. doi:http://dx.doi. org/10.1590/S1414-98932008000400012

Azevedo, L. A., Tatmatsu, D. I. B, \& Ribeiro, P. H. R. (2011). Formação em psicologia ea apropriação do enfoque da atenção primária à saúde em Fortaleza, Ceará. Trabalho, Educação e Saúde, 9(2), 241-264. doi:http://dx.doi.org/10.1590/\$1981-77462011000200005

Bardin, L. (2010). Análise de conteúdo. Lisboa: Edições 70.

Biscarde, D. G. dos S., Pereira-Santos, M., \& Silva, L. B. (2014). Formação em saúde, extensão universitária e Sistema Único de Saúde (SUS): Conexões necessárias entre conhecimento e intervenção centradas na realidade e repercussões no processo formativo. Interface Comunicação, Saúde e Educação, 18(48), 177-186.

Bastos, A. V. B., Gondim, S. M. G., \& Borges-Andrade, J. E. (2010). O psicólogo brasileiro: Sua atuação e formação profissional. O que mudou nestas últimas décadas? In O. H. Yamamoto, \& A. L. F. Costa (Orgs.), Escritos sobre a profissão de psicólogo no Brasil (pp. 255-271). Natal, RN: EDUFRN. doi:http://dx.doi.org/10.1590/1807-57622013.0586

Böing, E., \& Crepaldi, M. A. (2010). O psicólogo na atenção básica: Uma incursão pelas políticas públicas de saúde brasileiras. Psicologia: Ciência e Profissão, 30(3), 634-649. doi:http://dx.doi.org/10.1590/S1414-98932010000300014 
Ceccim, R. B., \& Carvalho, Y. M. de. (2005). Ensino da saúde como projeto da integralidade: A educação dos profissionais de saúde no SUS. In R. Pinheiro, R. B. Ceccim, \& R. A. de Mattos (Orgs.), Ensinar saúde: A integralidade e o SUS nos cursos de graduação na área da saúde. Rio de Janeiro: IMS/UERJ: ABRASCO.

Ceccim, R. B., \& Feuerwerker, L. C. M. (2004). O quadrilátero da formação para a área da saúde: Ensino, gestão, atenção e controle social. Physis, 14(1), 41-65.

Cezar, P. K., Rodrigues, P. M., \& Arpini, D. M. (2015). A Psicologia na Estratégia de Saúde da Família: Vivências da Residência Multiprofissional. Psicologia: Ciência e Profissão, 35(1), 211-224. doi:http://dx.doi.org/10.1590/1982-3703000012014

Conselho Federal de Psicologia. (2010). Práticas profissionais de psicólogos e psicólogas na atenção básica à saúde. Brasília, DF: Conselho Federal de Psicologia.

Davi, R. S., Dominguez, A. R., Araújo, M. V., \& Franco, E. M. (2016). O psicólogo nas unidades básicas de saúde: Relatos distantes do SUS. Actualidades em Psicologia, 30(120), 71-83. doi:http://dx.doi.org/10.15517/ap.v30i120.19836

Dimenstein, M.(2001).Opsicólogoeocompromissosocialnocontextodasaúdecoletiva. Psicologia em Estudo, 6(2), 57-63. doi:http://dx.doi.org/10.1590/\$1413-73722001000200008

Dimenstein, M., \& Macedo, J. P. (2010). Desafios para o fortalecimento da Psicologia no SUS: A produção referente à formação e inserção profissional. In M. J. P. Spink (Org.), A Psicologia em diálogo com o SUS: Prática profissional e produção acadêmica (pp. 207-232). São Paulo: Casa do Psicólogo.

Frank, J. R., Snell, L., \& Sherbino J. E. (2015). CanMEDs 2015: Physician Competency Framework. Ottawa: Royal College of Physicians and Surgeons of Canada.

Ferreira, J. L., Neto (2011). Psicologia, políticas públicas e o SUS. São Paulo: Escuta.

Gaskell, G. (2005). Entrevistas individuais e grupais. In M. Bauer, \& G. Gaskell (Orgs.), Pesquisa qualitativa com texto, imagem e som (pp. 64-89). Petrópolis: Vozes.

Lei n. 11.129, de 30 de junho de 2005 (2005). Institui o Programa Nacional de Inclusão de Jovens - ProJovem; cria o Conselho Nacional da Juventude - CNJ e a Secretaria Nacional da Juventude, altera as Leis no s 10.683, de 28 de maio de 2003, e 10.429, de 24 de abril de 2002; e dá outras providências. Brasília-DF: Presidência da República. Recuperado de http://www.planalto.gov.br/ccivil_03/_Ato2004-2006/2005/Lei/L11129.htm

Lima, N. T. (2015). Residências são uma modalidade diferenciada de formação [Entrevista concedida à Antonio Fuchs]. Recuperado de https://agencia.fiocruz.br/ residencias-são-uma-modalidade-diferenciada-de-formação

Minayo, M. C. de S. (2006). O desafio do conhecimento: Pesquisa qualitativa em saúde (9a ed.). São Paulo: Hucitec.

Minayo, M. C. de S. (2014). O desafio do conhecimento: Pesquisa qualitativa em saúde (13a ed.). São Paulo: Hucitec.

Nepomuceno, L. B. (2014). A construção social da prática psicológica na atenção primária à saúde do SUS. (Tese de Doutorado, Universidade Federal do Ceará, Fortaleza, CE).

Parisi, L. P., \& De Antoni, C. (2014). A psicologia na atenção primária à saúde: Práticas psicossociais, interdisciplinaridade e intersetorialidade. Clínica \& Cultura, 3(1), 71-85.

Pasini, V. L. (2010). Residência Multiprofissional em Saúde: De aventura quixotesca à política de formação de profissionais para o SUS. (Tese de Doutorado, Pontifícia Universidade Católica do Rio Grande do Sul, Porto Alegre, RS). 
Reis, C. dos, \& Guareschi, N. M. de F. (2013). A formação em Psicologia: Desafios para a inserção profissional nas políticas públicas de saúde. In A. M. C. Ximendes, C. dos Reis, \& R. W. de Oliveira (Orgs.), Entre Garantia de Direitos e Práticas Libertárias. Porto Alegre: Conselho Regional de Psicologia do Rio Grande do Sul.

Resolução n. 2, de 13 de abril de 2012 (2012). Dispõe sobre Diretrizes Gerais para os Programas de Residência Multiprofissional e em Profissional de Saúde. Brasília-DF: Secretaria de Educação Superior. Recuperado de

http://portal.mec.gov.br/index.php?option=com_docman\&view=download\&alias=15448resol-cnrms-n2-13abril-2012\&Itemid=30192

Resolução n. 466, de 12 de dezembro de 2012 (2012). Dispõe sobre as diretrizes e normas regulamentadoras de pesquisa envolvendo seres humanos. Brasília-DF: Conselho Nacional de Saúde. Recuperado de http://bvsms.saude.gov.br/bvs/saudelegis/cns/2013/ res0466_12_12_2012.html

Resolução n. 510, de 07 de abril de 2016 (2016). Dispõe sobre as normas aplicáveis a pesquisas em Ciências Humanas e Sociais. Brasília-DF: Conselho Nacional de Saúde. Recuperado de http://conselho.saude.gov.br/resolucoes/2016/Reso510.pdf

Resolução n. 597, de 13 de setembro de 2018 (2018). Dispõe sobre as recomendações do Conselho Nacional de Saúde à proposta de Diretrizes Curriculares Nacionais do curso de graduação em Psicologia. Brasília-DF: Ministério da Saúde. Recuperado de

http://conselho.saude.gov.br/resolucoes/2018/Reso597.pdf

Rocha, M. B., Almeida, M. M. S., \& Ferreira, B. O. (2016). Possibilidades de atuação profissional do psicólogo no âmbito da Atenção Básica em Saúde. Revista Brasileira de Promoção da Saúde, 29(1), 117-123.

Rodrigues, P. M., Paraboni, P., Arpini, D. M., Brandolt, C. R., Lima, J. V., \& Cezar, P. K. (2017). O registro em prontuário coletivo no trabalho do psicólogo na Estratégia de Saúde da Família. Estudos de Psicologia, 22(2), 195-202. doi:http://dx.doi. org/10.22491/1678-4669.20170020

Romagnoli, R. C. (2006). A Formação dos Psicólogos e a Saúde Pública. Pesquisas e Práticas Psicossociais, 1(2), 1-15.

Rosa, N. B., \& Silva-Roosli, A. C. B (2019). A Psicologia na Atenção Básica: Possibilidades de Intervenção na Promoção e Prevenção à Saúde. Revista Psicologia \& Saúde, 11(2), 99-114.

Silva, C. de A., \& Yamamoto, O. H. (2013). As Políticas Sociais na Formação Graduada do Psicólogo no Piauí. Psicologia: Ciência e Profissão, 33(4), 824-839. doi:http://dx.doi. org/10.1590/S1414-98932013000400005

Silva, R. B., \& Carvalhaes, F. F. (2016). Psicologia e Políticas Públicas: Impasses e reinvenções. Psicologia \& Sociedade, 28(2), 247-256. doi:http://dx.doi. org/10.1590/1807-03102016v28n2p247

Soares, T. C. (2005). "A Vida é Mais Forte do que as Teorias" O Psicólogo nos Serviços de Atenção Primária à Saúde. Psicologia: Ciência e Profissão, 25(4), 590-601. doi:http:// dx.doi.org/10.1590/S1414-98932005000400008

Spink, M. J., \& Matta, G. C. (2010). A prática profissional Psi na saúde pública: Configurações históricas e desafios contemporâneos. In M. J. P. Spink (Org.), A Psicologia em diálogo com o SUS: Prática profissional e produção acadêmica (pp. 25-51). São Paulo: Casa do Psicólogo. 
Starfield, B. (2002). Atenção primária: Equilíbrio entre necessidades de saúde, serviços e tecnologia. Brasília: Organização das Nações Unidas para a Educação, a Ciência e a Cultura. Yamamoto, O. H. (2003). Questão social e políticas públicas: Revendo o compromisso da psicologia. In A. M. B. Bock (Org.), Psicologia e o compromisso social (pp. 37-53). São Paulo: Cortez.

Recebido em: 02/09/2019 última revisão: 20/03/2020

Aceite final: 26/03/2020

\section{Sobre as autoras:}

Patrícia Matte Rodrigues: Mestra em Psicologia da Saúde pela Universidade Federal de Santa Maria (UFSM). Especialista em Residência Multiprofissional Integrada em Sistema Público de Saúde, com ênfase em Atenção Básica/Saúde da Família pela UFSM. Atualmente é psicóloga na Secretaria da Saúde do município de Garibaldi, Rio Grande do Sul (RS).E-mail: patricia_mr@yahoo.com.br, Orcid: https://orcid.org/0000-0003-1426-8166

Dorian Mônica Arpini: Doutora em Psicologia Social pela Pontifícia Universidade Católica de São Paulo (PUC). Mestra em Educação Brasileira pela Universidade Federal de Santa Maria (UFSM). Atualmente é professora titular aposentada e professora colaboradora do programa de pós-graduação em Psicologia na UFSM. E-mail: monica.arpini@gmail.com, Orcid: https://orcid.org/0000-0002-1667-5112

Camila Almeida Kostulski: Doutoranda em Psicologia pela Universidade Federal de Santa Maria (UFSM). Mestra em Psicologia da Saúde pela UFSM. Especialista em Direito de Família e Mediação de Conflitos pela Faculdade Palotina de Santa Maria (FAPAS). Bolsista CAPES. E-mail: camila.ak@hotmail.com, Orcid: https://orcid.org/0000-0003-3677-0501 